\title{
Climate mitigation scenarios with persistent COVID-19-related energy demand changes
}

\author{
Jarmo S. Kikstra $\mathbb{D}^{1,2,3,9 凶}$, Adriano Vinca ${ }^{1,4,9}$, Francesco Lovat ${ }^{1}$, Benigna Boza-Kiss $\mathbb{1}{ }^{1,5}$, \\ Bas van Ruijven ${ }^{1}$ 1, Charlie Wilson ${ }^{1,6}$, Joeri Rogelj ${ }^{1,2,3}$, Behnam Zakeri ${ }^{1,7}$, Oliver Fricko' and \\ Keywan Riahi ${ }^{1,8}$
}

\begin{abstract}
The COVID-19 pandemic caused radical temporary breaks with past energy use trends. How post-pandemic recovery will impact the longer-term energy transition is unclear. Here we present a set of global COVID-19 shock-and-recovery scenarios that systematically explore the effect of demand changes persisting. Our pathways project final energy demand reductions of 1-36 EJ yr ${ }^{-1}$ by 2025 and cumulative $\mathrm{CO}_{2}$ emission reductions of 14-45 $\mathrm{GtCO}_{2}$ by 2030. Uncertainty ranges depend on the depth and duration of the economic downturn and demand-side changes. Recovering from the pandemic with energy-efficient practices embedded in new patterns of travel, work, consumption and production reduces climate mitigation challenges. A low energy demand recovery reduces carbon prices for a $1.5^{\circ} \mathrm{C}$-consistent pathway by $19 \%$, lowers energy supply investments until 2030 by US $\$ 1.8$ trillion and softens the pressure to rapidly upscale renewable energy technologies.
\end{abstract}

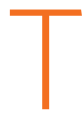
he ongoing coronavirus disease 2019 (COVID-19) pandemic has a far-reaching impact on society, with different repercussions across countries worldwide. The containment measures to limit the spread of the virus have resulted in reduced business activities, an increase in unemployment, travel restrictions, gathering limitations and changes in manufacturing and trade, affecting both the economy and people's daily lives ${ }^{1-3}$. As a consequence, people have had to temporarily change their lifestyles drastically, leading to changes in society's demand for energy on a daily basis ${ }^{1,4,5}$, which in turn has led to immediate observable effects on air quality, energy demand and greenhouse gas emissions, with several studies estimating the impact of restrictions on global $\mathrm{CO}_{2}$ emissions ${ }^{4,6,7}$. Although the global drop in greenhouse gas emissions in 2020 is very likely the largest on record in a single year ${ }^{8}$, temporary short-term reductions will not avert global temperature rise unless they are followed by long-term structural changes in energy systems $s^{4,9}$.

Governments have proposed and implemented major fiscal stimulus packages to help economies recover from this ongoing crisis. This has created a widely discussed opportunity for a 'green' and climate-positive recovery towards a net-zero emissions future ${ }^{10}$. Recent research has shown that policy support for decarbonization efforts in energy and transport is expected to increase ${ }^{11}$ and has identified policies for positive climate and economic recoveries ${ }^{12}$. However, in part due to the complexity of socially driven change, energy-economy modelling research has not yet focused on assessing the potential impacts of demand-side effects on climate mitigation challenges ${ }^{13,14}$.

In this study, we assess the potential effect of COVID-19-induced impacts on energy demand through recovery scenarios that vary the persistence of changes observed over the past year. We contribute a quantitative global analysis of how the near-term COVID-19 shock and alternative medium-term recovery pathways of demand-side changes could affect long-term energy demand. We find that enabling a low energy demand recovery can help reduce the costs of meeting Paris Agreement climate targets.

\section{An extreme event in a long-term model framework}

Assessing the effects of drastic near-term changes over the medium-to-longer term is challenging because it requires holistic treatment of both temporary and structural socio-economic changes that together define a set of alternative future pathways ${ }^{15,16}$. Recent studies have mostly assessed the observed impacts of lockdown measures in some western countries on the energy sector and $\mathrm{CO}_{2}$ emissions $^{6}$ and have tried to project trends for the coming decades following the 2020 shock $^{4,15}$. Some studies have focused on specific sectors like mobility ${ }^{17}$ or the power ${ }^{18}$ sector. Other studies ${ }^{16,19}$ have modelled links between current economic recessions and future projections of $\mathrm{CO}_{2}$ emissions, at the country level. Such studies do not explicitly consider different levels of persisting demand-side changes with feedbacks in an integrated energy-economy analysis.

Here, we combine a detailed bottom-up assessment of reported changes in energy services and energy demand in 2020 with macroeconomic modelling of sectoral changes driven by economic factors. First, we compare activity levels and energy service use intensities during the 2020 shock with historical data in three key sectors: transport, buildings and industry. Our focus is on social, behavioural, business model and infrastructural changes associated with COVID-19 restrictions. We then systematically evaluate medium-term uncertainties through a scenario design that illustrates distinct recovery pathways. These include regionally heterogeneous economic responses of varying intensities (through a gross domestic product (GDP) sensitivity analysis).

We construct possible recovery scenarios that either seize opportunities towards a new normality or revert to energy system

IInternational Institute for Applied Systems Analysis (IIASA), Laxenburg, Austria. ${ }^{2}$ Grantham Institute for Climate Change and the Environment, Imperial College London, London, UK. ${ }^{3}$ Centre for Environmental Policy, Imperial College London, London, UK. ${ }^{4}$ Institute for Integrated Energy Systems, University of Victoria, Victoria, British Columbia, Canada. ${ }^{5}$ Central European University (CEU), Budapest, Hungary. ${ }^{6}$ Tyndall Centre for Climate Change Research, University of East Anglia (UEA), Norwich, UK. ${ }^{7}$ Sustainable Energy Planning, Aalborg University, Aalborg, Denmark. ${ }^{8}$ Graz University of Technology, Graz, Austria. ${ }^{9}$ These authors contributed equally: Jarmo S. Kikstra, Adriano Vinca. e-mail: kikstra@iiasa.ac.at 
Table 1 | Sectoral elements of four scenario narratives

\begin{tabular}{|c|c|c|c|}
\hline & Transport & Buildings & Industry \\
\hline Restore & $\begin{array}{l}\text { Return to pre-pandemic levels. Shares } \\
\text { of private transport, vehicle ownership } \\
\text { and international aviation activity are } \\
\text { restored. }\end{array}$ & $\begin{array}{l}\text { Return to pre-pandemic levels of private } \\
\text { and public space usage in terms of size, } \\
\text { intensity and location. }\end{array}$ & $\begin{array}{l}\text { Industrial activity, production levels and supply } \\
\text { chain structures return to pre-pandemic levels, } \\
\text { coupled with the reduction in economic activity. }\end{array}$ \\
\hline Self-reliance & $\begin{array}{l}\text { Concerns about health risks remain for } \\
\text { a longer time. Shift to private transport } \\
\text { is combined with pre-pandemic } \\
\text { teleworking levels, leading to a strongly } \\
\text { muted overall increase in public transit } \\
(+3 \%) \text { while car and two-wheeler } \\
\text { usage surges (+24\%). Air travel is } \\
\text { high (+11\%). Freight activity nearly } \\
\text { fully recovers, but to lower levels than } \\
\text { counterfactual projections due to the } \\
\text { persistence of the economic shock. }\end{array}$ & $\begin{array}{l}\text { Health risk considerations and persistent } \\
\text { social distancing behaviour mean } \\
\text { total utilization rate }\left(\mathrm{m}^{2} \text {.degree-days }\right) \\
\text { increases }(+11 \% \text { globally, }+12 \% \text { in } \\
\text { residential, }+9 \% \text { in non-residential } \\
\text { buildings). Both home usage (office, } \\
\text { school, online services and e-retail) } \\
\text { and shares of under-occupied but } \\
\text { temperature-controlled non-residential } \\
\text { space increase, leading to some } \\
\text { duplication of personal space. }\end{array}$ & $\begin{array}{l}\text { Growth in private space (living, working and } \\
\text { travel) and new business solutions (online, thus } \\
\text { delivery and packaging), a legacy of duplication } \\
\text { of industrial sourcing (glocalization). The largest } \\
\text { effect is seen in demand for steel ( }+5-10 \% \text { ) due to } \\
\text { machinery demand, chemical and paper ( }+2-3 \%) \text {. } \\
\text { The combined effect with an economic recession } \\
\text { results in a global }+10 \% \text { activity compared with } \\
2019 \text {, which is } 4 \% \text { higher than it would be without } \\
\text { a pandemic. }\end{array}$ \\
\hline Green push & $\begin{array}{l}\text { The large reduction in commuting } \\
\text { trips and long-distance travel is highly } \\
\text { persistent }(-17 \% \text { aviation). Especially } \\
\text { in urban areas, private car use remains } \\
\text { low }(-7 \%) \text {. Transport needs are instead } \\
\text { fulfilled by rail }(+31 \%) \text { and road public } \\
\text { transport }(+23 \%) \text { in part enabled by } \\
\text { lower actual and perceived health risks } \\
\text { compared with other scenarios. }\end{array}$ & $\begin{array}{l}\text { Utilization rate of buildings increases } \\
(+7 \% \text { globally). Strong increase in the } \\
\text { use of thermally conditioned homes } \\
(+11 \%) \text { due to relocation of work, } \\
\text { services (schooling, retail, administration } \\
\text { and so on) and intensification of domestic } \\
\text { activities (cooking, entertainment and so } \\
\text { on). Some offsetting effect by reduction } \\
\text { and better use of non-residential space } \\
(-8 \%) \text { and efficiency gains due to user } \\
\text { behaviour and non-residential space } \\
\text { optimization. }\end{array}$ & $\begin{array}{l}\text { Increased efficiency in industries (as a legacy of the } \\
\text { pandemic, where industries worked under labour } \\
\text { and raw material shortage pressures). Rebalancing } \\
\text { between local production and imports. Lower } \\
\text { mobility and change in modal splits and building } \\
\text { activity reduce global demand for steel ( }-5 \% \text { ) and } \\
\text { aluminium (-3\%) compared with non-pandemic } \\
\text { situation. Impacts on building utilization also } \\
\text { moderate the increase of aluminium and cement. } \\
\text { Increased online shopping (packaging) and staying } \\
\text { at home (more hygiene) lead to changes in paper } \\
\text { and chemical demand and an overall increase in } \\
\text { material production (+2\%). }\end{array}$ \\
\hline
\end{tabular}

These elements represent potential avenues for reaching characteristic levels of persistence of sectoral changes in 2025, compared with 2019 levels. Indicated values are global aggregations of the estimation for the two macro-regions, Global North and Global South (see Supplementary Notes 1-5 for further details).

structures that existed before the pandemic. Each of the energy pathways is illustrated with a set of assumptions consistent with the persistence of activity and structural changes (Table 1 and Fig. 1). We use the MESSAGEix-GLOBIOM Integrated Assessment Model $(\mathrm{IAM})^{20}$ to capture global economy, energy and climate dynamics and feedbacks in the medium-to-long term, including regionally heterogeneous responses to the COVID-19 emergency. This integrated assessment of shock, recovery and long-term outcomes shows the conditions under which COVID-19 can have the strongest implications for climate change mitigation.

\section{Energy demand drop in 2020 and alternative recovery pathways}

Lockdowns and other pandemic measures have had major impacts on energy-related activity, mostly on international travel, commuting, use of space, e-commerce and use of technologies ${ }^{21}$. In turn, this has affected energy demand in the buildings, transport and industrial end-use sectors. We set out to understand the implications of these changes for sectoral energy demand as well as for structural changes regarding the types and amounts of energy services consumed in each sector (Methods and Supplementary Notes 1-5).
We assess the direct impact of COVID-19 on the use of residential and commercial floorspace, use of electric appliances, travel (by mode) and industrial output. We find global final energy demand in 2020 to be about $25 \mathrm{EJ}$ (6\%) lower than it would have been without the pandemic. Of that, $9 \mathrm{EJ}$ of reductions are attributable to industry (6\% sectoral reduction) and $20 \mathrm{EJ}$ to transport (17\% reduction). By contrast, the buildings sector shows a small increase in demand of $5 \mathrm{EJ}$ (3\% increase), as growth in residential energy use growth has been only partially offset by reductions in commercial and public building energy use (Fig. $2 \mathrm{a}-\mathrm{c})^{22,23}$. As a result of these observed changes, we estimate total $\mathrm{CO}_{2}$ emissions in 2020 to be around $7 \%$ or $3 \mathrm{Gt}$ lower than they would have been without the pandemic (Fig. $2 \mathrm{~d})$. This provides an independent estimate within the range of earlier estimates ${ }^{4,6,8}$.

How these observed near-term impacts on demand-related activity play out over the medium term to 2025 is highly uncertain. We construct and analyse four scenarios to systematically explore this uncertainty space using a branching point design. The first branching point distinguishes recovery pathways that move towards restoring pre-pandemic 'normality' from pathways that seize opportunities towards a 'new normality' (Fig. 1). The second branching 


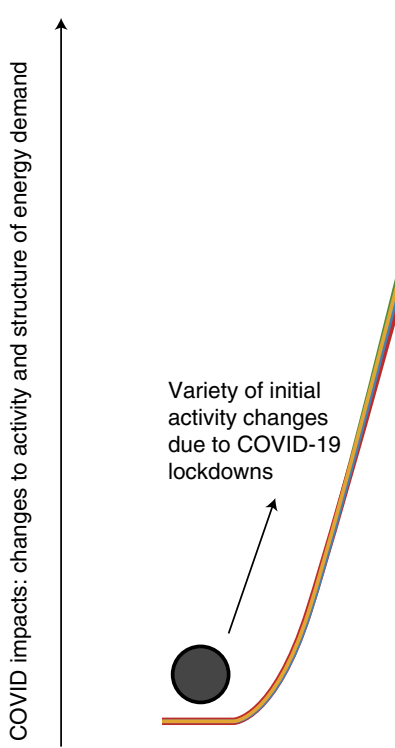

2019

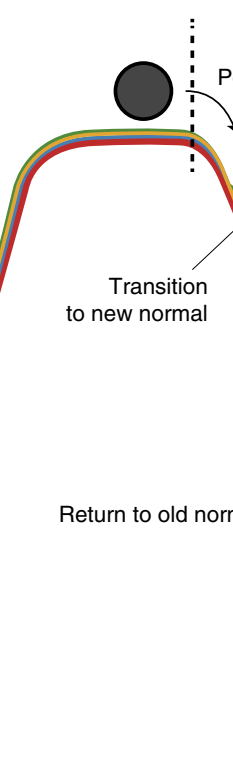

2020
Post-COVID-19 recovery

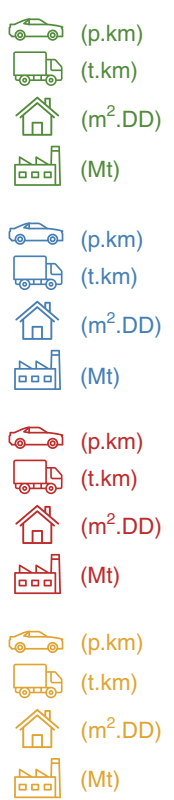

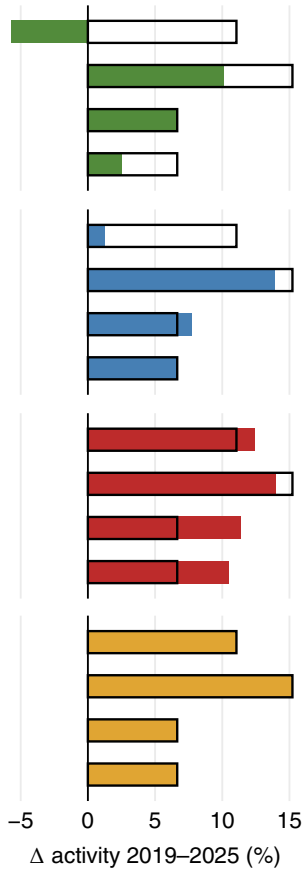

Fig. 1 Scenario design along the axis of COVID-related impacts. Note that the $y$ axis denotes disturbance compared with pre-pandemic 'normality' and not an increase in demand. Bar charts show relative changes in energy-related activity between 2019 and 2025 in passenger mobility (car icon), freight transport (truck icon), buildings (residential and non-residential; house icon) and industrial sectors (factory icon) for the four recovery pathways. The black outline boxes indicate the 2019-2025 change in the 'restore' scenario (yellow) and serve as a common reference point for the 'self-reliance', 'smart use' and 'green push' scenarios (red, blue and green, respectively). Indicators are passenger-kilometre (p.km), tonne-kilometre (t.km), metre squared-degree days ( $m^{2}$.DD) and material production in million tonnes (Mt). This image has been designed using resources from Freepik.com.

point distinguishes pathways with weaker or stronger responses to the demand-side changes experienced during lockdowns.

Table 1 summarizes the main elements of the four scenario narratives, along with the detailed assumptions about activity and structural changes in transport (modal shares), buildings (domestic-commercial-retail shares) and industry (production of different materials) over the period until 2025. The activity-structure-intensity methodology follows the approach of Grubler et al. ${ }^{24}$ (see Supplementary Notes 1-5 for full details). Economic uncertainty around GDP decline and recovery is further explored using sensitivity analysis with regional detail (Supplementary Note 6).

The restore and self-reliance scenarios describe recovery pathways characterized more strongly by path-dependence and system inertia. The restore scenario sees a strong return to pre-pandemic energy-related activity and structure. The self-reliance scenario comes with an amplified emphasis on individualism and national isolation, with somewhat reduced economic cooperation and social integration. Self-reliance implies increased use of private vehicles and larger working and home office spaces (Table 1).

The smart use and green push scenarios describe recovery pathways that learn from experiences during lockdowns. The smart use scenario sees positive experiences with enforced behavioural changes enduring over the medium term. For example, increased awareness of the impacts of air pollution, health and well-being benefits of less carbon-intensive transport and benefits of less commuting time and more teleworking become embedded in new social patterns affecting energy-related activity in both buildings and transport sectors (Fig. 1). The green push scenario illustrates strong learning supported by structures that enable enduring changes in active travel, digital substitution for physical transport, efforts to reduce health risks in public transport and directed downsizing of under-used retail and commercial buildings space. These distinct scenario narratives focus on the first- and second-order effects on the energy transition given the varying persistence of COVID19 impacts on energy demand. It is not the aim of this study to assess the dynamic of implementing specific policies, which would deserve a separate dedicated effort that also explicitly explores governance contexts.

Depending on the scenario, global energy demand surpasses 2019 levels between 2021 and 2023, with global final energy demand in 2025 remaining $1-36 \mathrm{EJ} \mathrm{yr}^{-1}$ lower than a counterfactual no-pandemic scenario and with different sectoral dynamics (Fig. $2 \mathrm{a}-\mathrm{c}$ ). The green push scenario is the only scenario to delay the rebound in energy demand considerably. Notably, the smart use scenario still sees a rebound of energy due to higher residential activity and recovering transport energy demand.

The range of final energy outcomes for the buildings and industry sectors across our four COVID-19 shock-and-recovery scenarios is relatively small compared with the full uncertainty range in future forecasts. By contrast, the range in transport final energy in 2025 due to COVID-19 recovery assumptions is almost four times as large as the projection uncertainties across five different IAM pathways simulating national policies without a pandemic (after harmonizing in 2020) (Fig. 2e). To get a sense of the magnitude of this change, transport energy demand reductions in the green push scenario by 2025 relative to a counterfactual no-COVID scenario are equivalent to a $12 \%$ reduction in global passenger transport activity (holding constant modal shares and fuel efficiencies). Alternatively, similar levels of energy demand reductions could have been achieved by shifting $18 \%$ of private transport activity to public transport or by electrifying approximately a third of global private road transport activity (if change were globally uniform).

Global $\mathrm{CO}_{2}$ emissions follow a similar trend, but pre-pandemic levels are reached between 2023 and 2033 depending more strongly on the recovery pathway (Fig. 2e). The cumulative carbon reduction is $14-45 \mathrm{GtCO}_{2}$ by 2030 compared with a counterfactual 
scenario without a pandemic. This reduction is attributable to the energy demand reductions in industry and transport, with the latter accounting for most of the variation between scenarios. Pre-pandemic, it was already clear that current climate action is inconsistent with the Paris Agreement's ambition of limiting global warming to well below $2{ }^{\circ} \mathrm{C}$ and pursuing the goal of limiting it to $1.5^{\circ} \mathrm{C}$ (ref. ${ }^{25}$ ). Our COVID-19 shock-and-recovery demand-side scenarios do not alter this picture, in the absence of additional stringent climate policies. The large economic uncertainty during the recovery has strong consequences for emission trends: rapid recoveries from economic recessions could more than offset emission reductions from the activity and structural changes in buildings, transport and industry (grey shaded area in Fig. 2d). Even with very strong reductions in global GDP, carbon budgets consistent with Paris Agreement goals will still be depleted fast (Fig. $2 \mathrm{f}-\mathrm{g}$ ). At most, it delays their depletion by 3 to 5 years (for $1.5^{\circ} \mathrm{C}$ and $2^{\circ} \mathrm{C}$, respectively) compared with a scenario without the pandemic. This emphasizes the continued importance of stringent and sustained climate policies alongside or as part of the economic recovery.

The strongest $\mathrm{CO}_{2}$ reductions are found in the Global North. Projected growth in energy and emission trends dominate the relatively small COVID-19 demand change effect in the Global South (see Supplementary Note 5.1 for more detail; region definitions are given in Supplementary Note 1).

\section{Energy transition challenges under alternative recoveries}

Across a diverse set of indicators, a lower-energy-demand green push recovery is found to have the lowest climate mitigation challenge (Fig. 3; more regional detail in Supplementary Figs. 19-26 and the online Scenario Explorer tool (https://data.ene.iiasa.ac.at/ engage/\#/workspaces/60)). Here we discuss the relative differences between scenarios pursuing a $1.5^{\circ} \mathrm{C}$ target, investigating the effects of missing opportunities to maintain parts of the energy reductions observed during the pandemic. A demand-side recovery from the pandemic that locks in high energy demand practices means that the system-wide post-recovery decarbonization rate has to be up to $3 \%$ faster over the period 2025-2040 (self-reliance scenario). The largest variation in decarbonization rates across scenarios is from transportation energy demand ( $4 \%$ for smart use scenario to $8 \%$ for self-reliance scenario with increased private vehicle use). Demand-side decarbonization rates for industry (3 to 5\%) and residential and commercial buildings ( 2 to $5 \%$ ) are slightly less dependent on the overall recovery (Fig. 3).

Pathways that aim to stabilize global temperatures around $1.5^{\circ} \mathrm{C}$ require considerable energy investments. Even if differences in the required pace of decarbonization are small, maintaining lower energy demand (as in the green push scenario) reduces energy investments until 2030 considerably. Compared with green push, letting energy demand restore to pre-pandemic structures instead means about 9\% higher investments (Fig. 3) or US\$1.8 trillion globally. The additional energy investments required for a self-reliance recovery that still meets the $1.5^{\circ} \mathrm{C}$ targets amount to US\$3.5 trillion (18\%). The potential missed opportunity for reducing energy investment needs is largest in the Global North (up to $21 \%$ for self-reliance). Similarly, the simulated aggregate carbon pricing until 2030 to meet the $1.5^{\circ} \mathrm{C}$ target is notably higher for the self-reliance (15\%) and restore (19\%) scenarios compared with green push (Fig. 3). Thus, if the post-COVID-19 recovery fails to embed low-carbon activity and structural change, economic incentives to decarbonize the system must be markedly stronger, particularly in the Global North, due to the larger impact of COVID-19 on activity, energy and emissions compared with the Global South (Supplementary Note 5.1).

Increased near-term transport energy demand forces transport electrification to be faster to meet the $1.5^{\circ} \mathrm{C}$ climate target. Electricity in transport in 2030 accounts for $9.5 \mathrm{EJ} \mathrm{yr}^{-1}$ in the green push scenario ( $11 \%$ of transport final energy). In the self-reliance scenario, it is higher at $12 \mathrm{EJ} \mathrm{yr}^{-1}$ (12\% of transport final energy). These noteworthy differences in the relative speed of electrification in Fig. 3 show the greater electrification challenge for transport if passenger mobility recovers from the COVID-19 shock mostly in the form of private vehicle use, increasing transport electricity from its 2019 value of $1.7 \mathrm{EJ} \mathrm{yr}^{-1}$. Failing to push for a green recovery that includes modal shift risks increasing the electrification challenge by on the order of 13 trillion electric vehicle (EV) kilometres extra per year by 2030 or about eight times the 2019 global electricity demand from $\mathrm{EVs}^{26}$.

A low-carbon energy transition requires strong decarbonization of the energy supply as well. Higher global energy demand means faster renewables growth to reduce emissions. Consequently, the share of electricity coming from wind and solar installations in 2030 could be more than 5\% higher in the self-reliance scenario than in the green push scenario, requiring a $10 \%$ faster upscaling of non-biomass renewable energy generation (Fig. 3). Regardless of the recovery pathway, the transitional challenge is large. Wind and solar electricity shares for 2030 range between $49 \%$ and $54 \%$ in our $1.5^{\circ} \mathrm{C}$ scenarios, up from $8 \%$ in 2019 .

Alongside rapid renewables deployment, rapid fossil fuel phase-out is another energy transition challenge. Lower energy demand in the near term is associated with faster phase-out of coal-fired power generation. This comes with potential near-term social challenges, though these are regionally heterogeneous due to different coal plant characteristics (Fig. 3). None of the presented scenarios with ambitious climate mitigation strategies towards $1.5^{\circ} \mathrm{C}$ see a recovery of coal use for energy after the steep reduction during the 2020 pandemic ${ }^{27}$.

\section{Medium-term green recovery yields climate mitigation benefits}

Most scenarios that aim to limit global warming to $1.5^{\circ} \mathrm{C}$ show global net-zero $\mathrm{CO}_{2}$ emissions around 2050 (ref. ${ }^{28}$ ). This requires fast and continued emission reductions through the decarbonization of energy systems. The pre-pandemic global emission level of about $42 \mathrm{GtCO}_{2} \mathrm{yr}^{-1}\left(\right.$ ref. $^{8}$ ), which was still trending upwards, would leave less than 10 years before closing the door on limiting temperature increase to $1.5^{\circ} \mathrm{C}$ (refs. ${ }^{25,29}$ ).

Fig. 2 | Final energy and emissions pathways under alternative COVID-19 recovery scenarios and their combination with economic uncertainty. a-c, Final energy use for the buildings (a), transport (b) and industry (c) sectors. d, Total annual $\mathrm{CO}_{2}$ emissions, including national determined contributions (NDCs), national policies from CD-LINKS, and scenarios consistent with $1.5^{\circ} \mathrm{C}$ with low or no temperature overshoot (OS) from Huppmann et al..$^{57}$. Vertical bars indicate the range of annual $\mathrm{CO}_{2}$ emissions in 2035 within a group of scenarios. e, Sectoral final energy in 2025 compared with five global IAM pathways simulating national policies. A, AIM/CGE 2.1; I, IMAGE 3.0.1; P, POLES CD-LINKS; R, REMIND-MAgPIE 1.7-3.0; W, WITCH-GLOBIOM 4.4; for scenario CD-LINKS_NPi. National policies are harmonized to the 2020 values in 'Baseline-no-COVID' using a constant offset. $\mathbf{f}$, Cumulative CO 2 emissions starting from 2019, with global $\mathrm{CO}_{2}$ budgets (black lines) visualized as reported in SR 15 for limiting peak warming to $1.5^{\circ} \mathrm{C}$ and $2{ }^{\circ} \mathrm{C}$ with $50 \%$ and $66 \%$ likelihood, respectively, while accounting for the assessed impact of Earth system feedbacks such as thawing permafrost. The range (yellow shading) surrounding these values represents non- $\mathrm{CO}_{2}$ scenario uncertainty. $\mathbf{g}$, Global GDP (market exchange rates) indexed to 2019 levels for our scenarios (thick black line), the used no-pandemic projection (dashed line) and uncertainty range (thin black line and grey shading). In all panels, grey shading shows the sensitivity range considering GDP uncertainty (see Supplementary Note 6 for more details). 
Our study confirms that the direct effect of the COVID-19 pandemic lockdowns on global emissions is negligible in the context of this challenge. The effects of the persistence of COVID-19-related energy demand changes alone (14-45 $\mathrm{GtCO}_{2}$ less by $2030 \mathrm{com}-$ pared with scenarios pre-COVID-19) are not nearly sufficient to meet emissions reduction targets, which require more fundamental structural changes in the energy system. This finding still stands when accounting for economic uncertainty, even considering a very long economic downturn paired with lower emissions. Additionally, we calculate that if the energy demand recovery pathways were

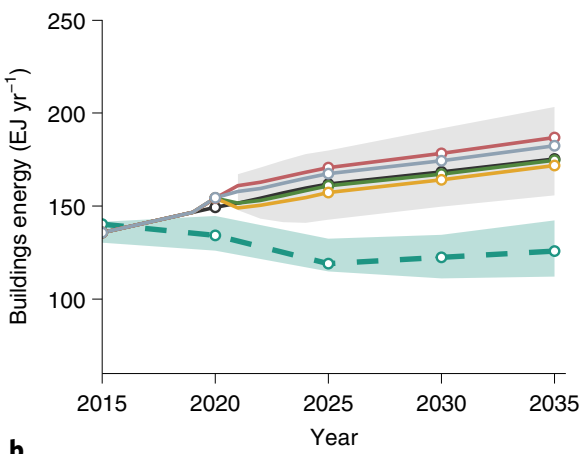

b

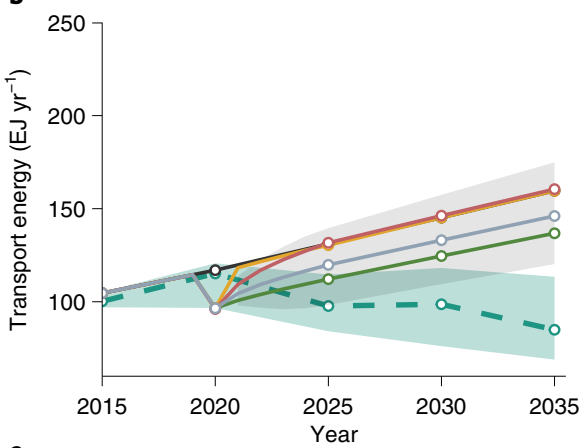

c

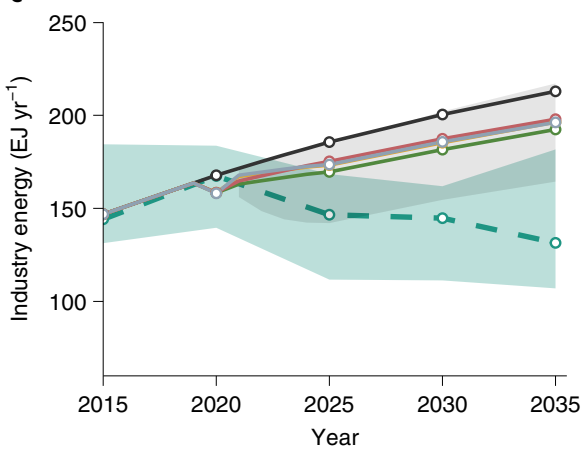

e

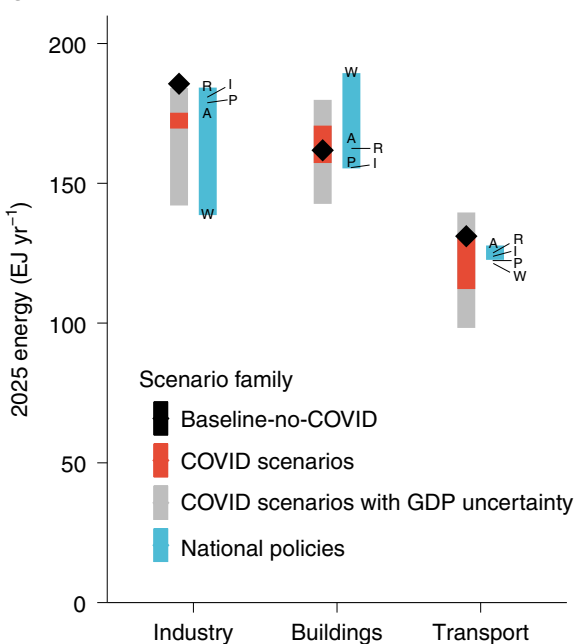

d
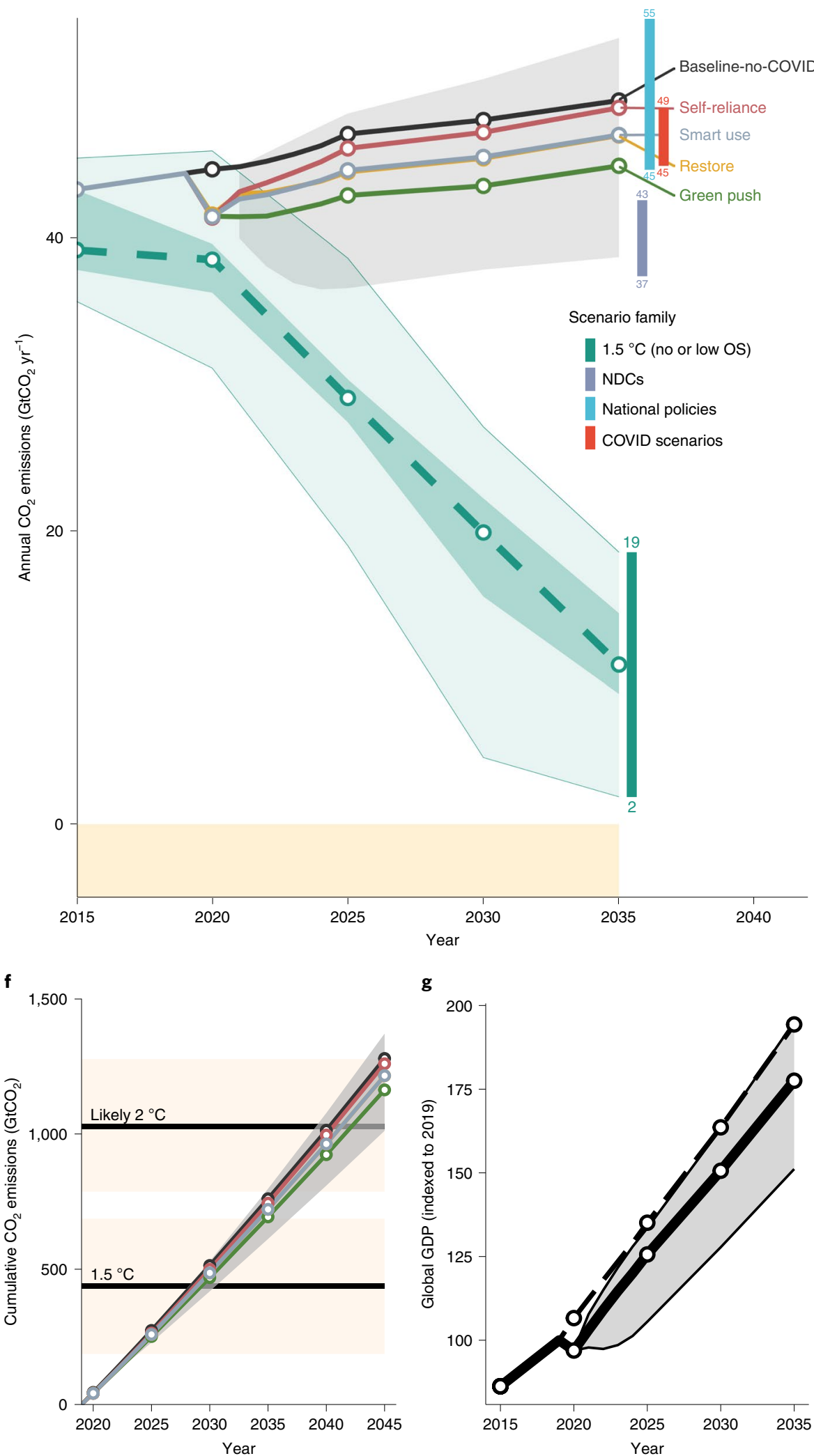

g

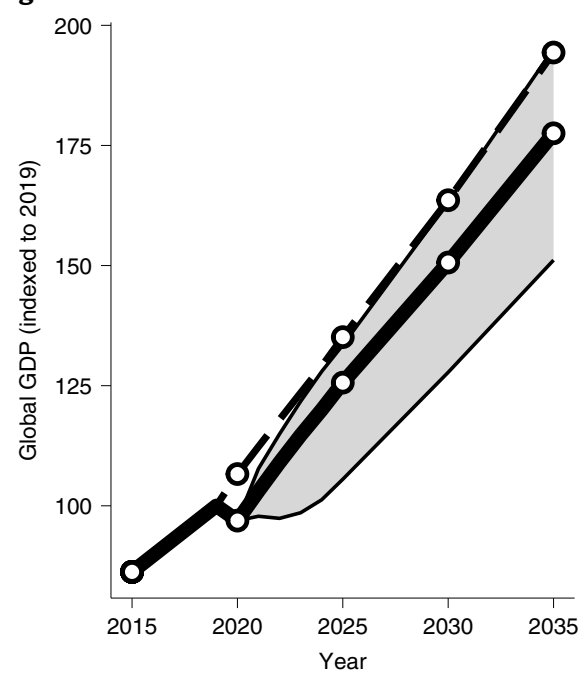




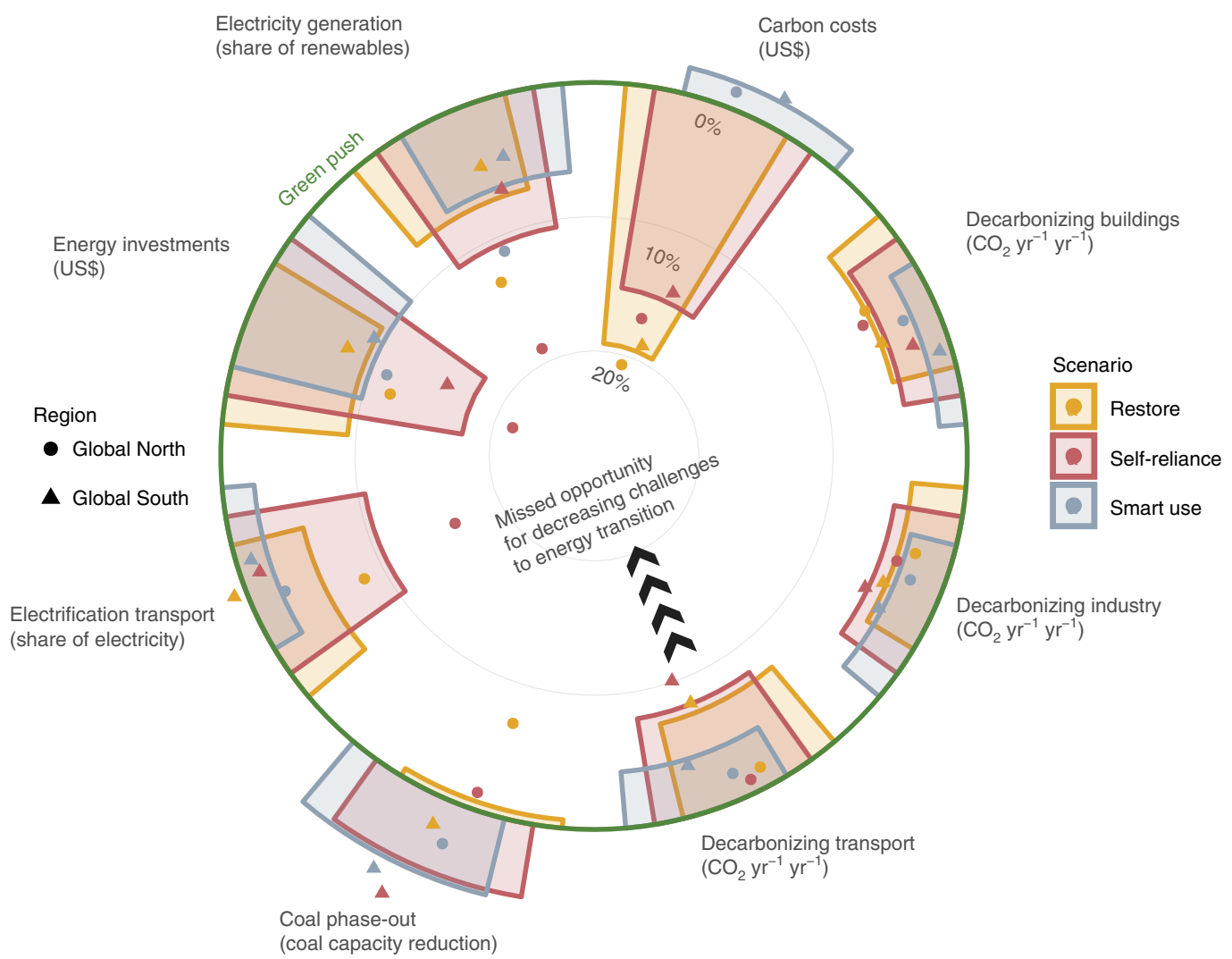

Fig. 3 | Alternative medium-term recovery pathways affect the size of the energy transition challenge for limiting global warming to $1.5^{\circ} \mathrm{C}$. Each wedge shows the global per cent variation in a specific indicator of mitigation effort required in the restore (yellow), self-reliance (red) and smart use (grey) scenarios relative to the scenario with the lowest transition challenges (green push, green); points and triangles refer to Global North and South results (Supplementary Note 5.1). Electricity generation: the share of solar and wind in electricity generation. Carbon costs: the net present value of the global carbon price multiplied by annual greenhouse gas emissions for the period 2020-2030. Decarbonizing buildings, industry and transport: increase in post-recovery decarbonization pace in 2025-2040 compared with the reference scenario under the same climate target. Coal phase-out: reduction in cumulative coal energy production capacity 2020-2030. Electrification transport: share of electricity of transport energy in 2030. Energy investments: cumulative energy supply investments 2020-2030.

combined with an equal carbon price trajectory consistent with the $1.5^{\circ} \mathrm{C}$ target, a green push recovery could avoid another $24 \mathrm{GtCO}_{2}$, compared with the restore scenario.

Because of the urgent need for strong $\mathrm{CO}_{2}$ emission reductions, seizing opportunities for maintaining energy demand changes (green push) can increase the probability of staying below $1.5^{\circ} \mathrm{C}$, reducing the cost of similar emissions abatement. Conversely, a recovery pathway with higher energy demand means further efforts are needed by 2030 to achieve an additional $2.5 \mathrm{EJ} \mathrm{yr}^{-1}$ electricity for transport, an additional 5\% electricity generation share from wind and solar and investment of an additional US\$3.5 trillion. These additional efforts are on top of already highly ambitious decarbonization needs. We also find these comparative differences between scenarios to be robust for different climate mitigation goals (see Supplementary Fig. 25 for comparison with the wider scenario literature).

\section{Insights for an energy demand recovery}

It is important to understand to what extent different behavioural and structural changes drive emissions or enable emissions reductions. We have shown the implications of four alternative internally consistent pathways of energy demand recovery from the COVID19 shock and have quantified first-order effects of demand-side changes in each pathway.

The full sectoral contributions to $\mathrm{CO}_{2}$ emissions savings from demand-side changes include both direct end-use emissions and indirect effects on emissions in manufacturing, supply chains and production. For industry, these upstream effects of energy demand reduction are a much larger portion of the change than for transport $\mathrm{CO}_{2}$ change, which is mostly related to direct energy end-use (Supplemental Note 5.3). Even after accounting for upstream effects, the $\mathrm{CO}_{2}$ emissions savings that could persist related to the pandemic are predominantly transport related. Full transport $\mathrm{CO}_{2}$ reductions by 2025 in the green push scenario without additional climate policies would amount to about $9 \%$ of the emission reductions in a restore $1.5^{\circ} \mathrm{C}$-consistent pathway. Looking at relative emission changes in sectors when switching from the restore to the green push scenario further illustrates the relative importance of the transport sector. Between the two scenarios, increased emissions related to residential and commercial buildings are about an order of magnitude less than transport-related reductions. Emissions reductions in the transport sector are also about four times larger than in the industry sector. This relative difference in emissions savings increases to eight times when additionally applying a stringent $1.5^{\circ} \mathrm{C}$-consistent climate constraint, illustrating that the persistence of transportation changes is key to the differences in mitigation challenges we report in our results, especially in the Global North, where higher shares of the workforce have the resources to change commuting habits.

There is no magic bullet for reaching Paris Climate Agreement goals. However, guiding post-pandemic recovery in energy-demand-related activities towards less energy-intensive 
activities is an important part of the arsenal. Supporting working from home and teleconferencing to reduce flying and commuting can have strongly beneficial outcomes for emissions, especially if combined with the rationalization and reduction of office space, other workspace, reduced administration (for example, public administration), entertainment, shopping spaces and intensities (as in the green push scenario). With online, delivery-based, less material-intensive alternatives becoming popular during the pandemic, reducing the carbon intensity of such services is important too, albeit not currently a dominant factor. In addition, enabling the shift to more active transport and more public transport under mitigated health and safety risks is important. Industrial supply chain rationalization and moderation of freight distances can help further decrease emissions. For more sectoral detail and intersectoral comparison of the magnitudes of change in terms of activity and energy intensity, see Supplementary Notes 2-5.

This study has systematically explored the consequences of persistent energy demand shifts for energy transition challenges, acknowledging large economic uncertainty. The insights from this study provide the background against which proposed recovery packages can be evaluated. Investigating potential additional path dependency of either intensifying or weakening structural changes related to shifts in lifestyles beyond 2025 could usefully expand on this work.

\section{Methods}

MESSAGEix-GLOBIOM. We use the MESSAGEix-GLOBIOM IAM ${ }^{30}$ to assess the implications of different COVID-19 scenarios on the energy system and derived indicators such as greenhouse gas emissions and energy investment needs.

MESSAGEix-GLOBIOM is a process-based IAM that allows for a detailed representation of the technical-engineering, socio-economic and biophysical processes in energy and land-use systems. It is a linear/mixed integer optimization model, aiming to satisfy exogenous and endogenous demands at least $\operatorname{cost}^{20}$. MESSAGEix-GLOBIOM includes a linkage to the energy system model and MACRO, a macroeconomic model, which maximizes the intertemporal utility function of a single representative producer-consumer in each world region. The optimization result is a sequence of optimal savings, investment and consumption decisions. The main variables of the MACRO model are the capital stock, available labour and energy inputs, so that the model can describe the feedback of end-use prices on demand for energy services ${ }^{31}$.

The linkage between energy and macroeconomic models is established through an iterative process. First, energy prices are calculated in MESSAGEix-GLOBIOM based on a reference exogenous energy demand data. Then, these energy prices are passed to MACRO, where energy demand is recalculated considering the impact of energy supply cost on a reference trajectory of GDP for each model region. In return, new energy demand data resulting from the MACRO solution are fed back to MESSAGEix-GLOBIOM, which influences the demand-supply balances, resulting in new energy prices. The iteration of energy prices and energy demand between the two models continues until the output of the two models converges to a stable trajectory within a predefined tolerance (more details can be found in Krey et al. ${ }^{30}$ ).

MESSAGEix-GLOBIOM has been widely used for the analysis of greenhouse gas emission pathways under a range of climate and socio-economic futures ${ }^{32,33}$, as well as in the assessment of climate mitigation strategies, including specific assessments of energy investment needs ${ }^{34,35}$. It has been one of the models informing global emission pathway analyses such as the reports of Intergovernmental Panel on Climate Change (IPCC) ${ }^{28}$, Global Energy Assessment $(\text { GEA })^{36}$ and the World in 2050 (ref. ${ }^{37}$ ). The global model version defines a set of 11 macroeconomic regions. The time horizon of the optimization framework goes from 2020 to 2100 , with a non-regular distribution of time steps. For this analysis, the model was extended to include individual years between 2020 and 2025, 5-year periods between 2025 and 2060 and 10-year periods between 2060 and 2100 . Compared with previous versions, the addition of the yearly periods $(2021,2022,2023$ and 2024) for this analysis crucially allows for a better focus on the short-term dynamics that are important for COVID-19 shock-and-recovery scenarios.

The socio-economic assumptions of MESSAGEix-GLOBIOM are based on the Shared Socioeconomic Pathways (SSPs) ${ }^{33,38}$, a set of internally consistent narratives and assumptions for main socio-economic drivers widely adopted and updated by the IAM community ${ }^{33}$. SSP2 is adopted as the starting point for this analysis ${ }^{32}$ because it is designed to extend historical trends.

The impact of COVID-19 on the economy is modelled based on external GDP estimates for 2020 and sees a 4 -year recovery to 'reference' growth rate values of SSP2 in the main scenarios. Energy demand reductions are a result of a bottom-up sectoral assessment both for the year 2020 and for four recovery scenarios. The model is first calibrated to fix the GDP and energy demand values in 2020 . The calibration results in two parameters, 'GDP growth rates' and 'autonomous energy efficiency improvements (AEEI)', which guarantee that the desired trend of GDP and energy demand in MACRO align with the exogenously defined values over time. The alternative energy demand pathways thus come with slightly different AEEI values. Further details on the calibration process can be found in Krey et al. ${ }^{30}$ and Messner and Schrattenholzer ${ }^{31}$.

Bottom-up assessment of 2020 shock on energy demand. The disruptive effect of the COVID-19 pandemic has had a direct impact on energy-using activities ${ }^{39,40}$. It has changed the activity, structure and intensity components of our mobility; how we use residential and public buildings and workspaces; and the production of goods and materials. The changes that we have taken into account are direct, or first-order, impacts induced by the COVID-19 pandemic itself and the containment measures, such as local and national lockdowns, distancing requirements, higher hygiene standards and restricted international trade and travel ${ }^{4,6}$. We also included second-order (indirect) effects of intersectoral changes. The energy demand shock before macroeconomic calibration was assessed using a bottom-up approach mostly independent from the economic downturn. We did this by assessing changes in activity and structure in three demand sectors: transport, buildings and industry. First, we collected data on observed demand shocks during the COVID-19 crisis in each of the sectors (data until December 2020, collection cut-off date March 2021). We mapped the 2020 data onto 2019 observations using a year-on-year method. Where no full-year data were available, we estimated 2020 values on a cluster of impact assessments taking into account the peak impacts. Then, we combined assessments of individual subsectoral activity reductions and aggregated them to calculate a total effect on global energy demand, extrapolated onto the spatial resolution of the MESSAGEix-GLOBIOM IAM ${ }^{20}$. A detailed description of the estimation of the 2020 energy demand shocks can be found in Supplementary Notes 2, 3 and 4 for the three demand sectors, respectively.

COVID-19 scenario framework. The recovery narratives in this study explore two principal uncertainties through a branching point design (Fig. 1), exploring potentially persistent changes related to the demand-side shock during the pandemic. The medium-term trends (2021-2025) use 2019 as a base year to compare changes with the pre-pandemic levels. Detailed narratives and quantitative assumptions for the transport, industry and buildings sectors are described in Transport, Industry and material production and Buildings, respectively, and in Supplementary Notes 2, 3 and 4, respectively. These scenarios are considered baseline scenarios that do not include explicit climate policy assumptions. Our modelling approach does not include the dynamic modelling of specific policy interventions such as the effects of the large-scale fiscal stimulus packages announced by many countries (see, for example, Andrijevic et al. ${ }^{10}$ ). Rather, the alternative scenarios assume different levels of persistence of COVID-19-related impacts that are plausibly linked with narratives of demand-related changes, such as lifestyle changes (teleworking, entertainment and travel routines) or business models (online health consultations) that could be induced or pertained through various packages of policies and that can have benefits for climate mitigation. These pathways are combined with carbon budgets to create combined COVID19-recovery and climate mitigation scenarios (Mitigation analysis).

\section{GDP pathways, coupling and sensitivities. Along with transformations in} energy service demand, the COVID-19 pandemic came with a major financial and economic crisis in 2020. To be able to clearly represent the different dynamics between the initial shock and the long-term response of the COVID-19 pandemic, we modelled both the economic shock in 2020 and the level of persistence of this economic shock in the short and long run. Considering the highly unpredictable nature of the current crisis, we deployed a maximally transparent, general-purpose framework to model possible macroeconomic effects of the COVID-19 pandemic.

Assessing the impact of COVID-19 on the economy in 2020 and after has been a challenge for economists, including the major financial institutes and central banks ${ }^{41}$. Consequently, initial, very uncertain estimates have been updated over time ${ }^{42,43}$. We captured this uncertainty by collecting a range of estimates of widely used economic prospects (including public entities, central banks and private rating agencies; Supplementary Note 6). Regional and national data from multiple sources were included to calculate the expected GDP shock for 2020 for the 11 modelled regions. From these sources, we estimated an average expected impact on the economy, as well as lower and higher estimates (the 10th and 90th percentiles of the sample, respectively). Supplementary Table 36 reports the regional values by source and the final values adopted in the model.

To acknowledge that the impacts on GDP levels are not restricted and are highly uncertain, we chose to systematically assess the sensitivity of the price-induced effect of a wide range of alternative GDP pathways. With a growth rate $g$, regional GDP levels develop following $\mathrm{GDP}_{r, t}=\mathrm{GDP}_{r, t-1}\left(1+g_{r, t}\right)$, where $r$ and $t$ stand for region and year, respectively. For projecting 2021 GDP levels, we apply a regional 1-year persistence parameter $\rho$ following $\mathrm{GDP}_{r, t}=\mathrm{GDP}_{r, t-1}\left(1+g_{r, t}-\rho_{r} \gamma_{r, t-1}\right)$, similarly to previous work ${ }^{44}$, where $\gamma$ represents an economic shock. The applied $\rho$ values are calculated based on the 
difference in GDP prospects in World Bank and International Monetary Fund (IMF) prospects before and after the COVID-19 crisis (Supplementary Note 6). Subsequently, to include both the long-term effect of the economic shock and the dynamics of the underlying SSP2 scenario, we let the GDP growth levels converge back linearly to the underlying growth rate.

In the quantification of the recovery scenarios, we treated the economic recovery and the energy demand trajectories independently, while allowing for macroeconomic feedbacks to energy demand shocks using the MESSAGE-MACRO iteration for each baseline calibration. We refrain from explicit exogenous coupling of GDP trajectories and energy scenarios because the nature of this crisis and its recovery are too uncertain to warrant such an approach. The main recovery scenarios discussed in the main text thus follow the same GDP recovery trajectory, with supplemental sensitivity runs based on varying the persistence parameter and the time it takes for growth rates to return to their originally projected values under SSP2.

Transport. We estimated the 2020 impacts on transport activity using a bottom-up assessment of the impact of the COVID-19 crisis on mobility, independent of the indirect effects of the GDP shock in 2020. The sharp decrease in transport activity in 2020 was mainly driven by the lockdown restrictions, which imposed a close-to-total halting of mobility for non-essential services ${ }^{39,40,45}$. We assumed a moderate shock across the existing estimates for each region and individual transport modes: rail; cars and two-wheelers; public transport (bus, tram and metro); aviation (domestic and international) and non-motorized transport for passengers and rail, road, international shipping and aviation for freight (detailed assumptions in Supplementary Note 2).

We used developments in five main elements as the starting point for the transport recovery scenarios: international tourism, commuting, business travel, online retail, use of mass transit and active mobility. In the restore scenario, no changes occur, and the recovery follows the patterns as foreseen under the SSP2 scenario. Under the self-reliance scenario, both international tourism and business travel revert to pre-COVID-19 levels, and commuting returns to pre-COVID-19 levels as well but is mostly car-bound. Online retailing sees a lower increase than in the other narratives. The use of public transport is sharply reduced, and active transport modes revert to pre-COVID-19 levels as well. In the smart use scenario, domestic tourism is rediscovered, and business trips are partially substituted by video conferencing. Partial teleworking remains common after the discovery of better work-life balance benefits and productivity levels. Increased adoption of online retail leads to an increase in road freight activity and reduced shopping trips. The use of mass transit is reduced: short-distance trips are replaced by non-motorized transport, while partial teleworking reduces the need for commuting. Finally, active mobility modes increase slightly as levels of usage during the pandemic are retained, driven by increased health benefits and perceived reduction of pollution levels. In the green push scenario, international tourism is reduced, and low-carbon modes dominate domestic travel. Business travel is strongly muted due to common video conferencing and discouraging policies. Commuting levels are reduced due to a high share of teleworking, and online retail is increasing. Targeted incentives lead people back to mass transport options, and investment in active mode infrastructure together with disincentivizing use of private cars sharply decreases the use of private transport modes.

These narratives were used to quantify transport-sector energy demand under each scenario (see the detailed description of the quantitative analysis and assumptions in Supplementary Note 2). We used the MESSAGEix-GLOBIOM SSP2 scenario as a starting point and combined the GDP projections in combination with the bottom-up scenario analysis to determine relative changes in energy intensity of transport as the joint effect of economic recovery and sectoral structural change.

Industry and material production. For the quantification of the energy demand and climate impact of the industry sector, we evaluated how the changes in 2020 in the activity, structure and intensity components of material production persist, which are directly impacted by the GDP shock. The pandemic changed total industrial production levels as well as production structures. Changes in individual lifestyles and institutional, social and commercial settings had a direct impact on industry ${ }^{46,47}$, and activity in industry was impacted indirectly as a result of changed demand in products in other sectors.

We used developments in a handful of driving elements as starting point for the industrial recovery scenarios: manufacturing activity, raw material availability, upstream sectors, labour markets, digitalization, individual mobility changes and construction and renovation changes. In the restore scenario, changes are driven by GDP, and recovery follows the patterns as foreseen under the SSP2 scenario. Under the self-reliance scenario, activity levels, structures and facility management aim to return to normal, but with extended purposes resulting from foreseeing new pandemics. Acquisition of raw materials is preferred from local sources due to nationalization and protectionism, and there is a focus on local storage ${ }^{48}$. Falling export markets and protection of home production and sales determine the demand for manufacturing products, while labour markets return to a pre-pandemic situation. Under this scenario, there is a lot of duplication of digital and offline solutions and increased hygiene, driving up material demands. In the smart use scenario, production repurposing and reduced activity due to process and material efficiencies inherited from the lockdown determine the level of activity. Raw materials are available, but transportation costs and risks of export availability are priced in. Digitalization and efficiency uptakes influence demand in primary sectors, and labour market reorganization reduces primary and secondary sector workers. Digitalization drives a moderate impact from online shopping, such as more packaging, more freight transport and more demand for electronics. Reduced overall transport demand impacts automobile production. In the green push scenario, manufacturing activity is driven by a thorough drive to increased process and material efficiencies. There is a focus on raw material efficiencies and on the balance between transportation and local solutions in light of sustainability. Upstream demand is driven by further increases in digitalization, efficiency and a focus on circular economy, while labour markets see financial and social support to adjust to a greener industry. There is further enhancement of digitalization impacts with policies towards efficiency improvements.

These narratives were used to quantify industry-sector energy demand under each scenario (see details and assumptions in Supplementary Note 3). We used the MESSAGEix-GLOBIOM SSP2 scenario as a starting point and combined the GDP projections in combination with the bottom-up scenario analysis to determine relative changes in energy intensity of industry as the joint effect of economic recovery and sectoral structural change.

Buildings. We used data on activity (floorspace) and energy intensity derived from the base-year information in Grubler et al. ${ }^{24}$ as the starting point for two global regions, Global North and Global South. We estimated the utilization factor of total space in the residential and the non-residential sectors in the base year (2019), expressed in floorspace.degree-days $\left(\mathrm{m}^{2} . \mathrm{DD}\right)$. This estimate is based on vacancy rates due to second homes, relocation, lack of tenants in the residential sector (see, for example, Huuhk $\mathrm{a}^{49}$ ) and closed but not yet sold business space ${ }^{50}$ as well as occupancy and thermoregulation rates (space and time) in homes, offices and retail (using O'Brien et al..$^{51}$ and $\mathrm{O}^{\prime}$ Brien and Yazdani Aliabadi ${ }^{52}$ ), in addition to assessing the additional energy demand for heating/cooling for longer occupancy ${ }^{51,52}$. We assumed changes in three dimensions: (1) change in total space due to additional construction, demolition or repurposing as a secondary effect, (2) change in the use factor of space respectively in the two subsectors and (3) the energy intensity of space demand in terms of thermal and electric energy demand.

In 2020, the impact on the total levels of activity (floorspace.DD) is a $2 \%$ increase compared with 2019 , because the decrease in the utilization of non-residential floorspace is compensated by increased in-home use. Region- and country-specific stringency of pandemic measures critically transforms the way buildings are used. A larger impact is observed in the Global North due to the dominance of hard lockdowns combined with incentives to stay at home, while typically less comprehensive and curfew-based measures are observed in the Global South

We determined the consequences of the pandemic-induced space reorganization in thermal and electric demand with a bottom-up approach, also in the medium term, reflecting the level of persistence of the behavioural, infrastructural and business model changes. The key drivers influencing behaviour and lifestyle change are the relocation of work and education and new business models for entertainment, socialization, administration, services and so on. There are important differences between the Global North and Global South, with the performance of emerging economies following yet a different trend.

In the restore scenario, none of the changes experienced in 2020 persist, and recovery follows the patterns as foreseen under the SSP2 scenario. The self-reliance scenario for buildings is characterized by the extension of distancing measures due to the persistence of higher health concerns and related distancing preference due to a fear of extended or new pandemics. In the Global North, teleworking persists at low levels but leads to duplication of digital and offline solutions and duplication of home offices and office buildings. Energy demand is high due to this duplication of buildings and a reversal of the sharing economy trends observed in past years. The emergence of secondary homes increases the average floor space per person. Homes are used intensively by being inhabited for more hours per day ${ }^{23}$, and the increased time spent at home increases energy demand for cooking, crafting, information and communication technology usage and entertainment.

In the smart use scenario, the building sector is characterized by a persistence of the transformation of building space for work, leisure, administration and services experienced during 2020. As the intensity of used floorspace remains unchanged, the energy intensity of total floorspace increases $(+1 \%$ compared with 2019 ) due to a higher use of residential buildings ( $+9 \%)$, which is not compensated by a similar reduction in commercial and public buildings ( $-2 \%$ use) because of increased idle floorspace. Intensity changes in the Global North and Global South are similar, in spite of the limited teleworking potential in the latter ${ }^{54}$, due to population increase and already high multipurpose use of buildings. In the green push scenario, the increase in activity and energy demand per floorspace in residential buildings ( +11 and $+5 \%$, respectively) as a result of the increased teleworking and other activities at home (cooking, crafting, entertainment and so on) is moderated by the changes in space utilization ( $-8 \%$ space use) and efficiency gains $(-4 \%$ intensity). This is achieved through a reduction of 
workspace for part-time teleworkers, reorganization of public space and the persistence of business model changes that emerged during the pandemic. These counterbalancing trends result in an overall net-zero change in building energy demand in 2025 compared with 2019.

The above narratives were used to quantify the energy demand changes with a bottom-up approach under each scenario and combined with the GDP projections based on the MESSAGEix-GLOBIOM SSP2 scenario to determine relative changes in final energy intensity of the building sector as the joint effect of economic recovery and sectoral structural change. For more detailed information, see Supplementary Note 4

Mitigation analysis. Besides middle-of-the-road reference scenarios, which do not assume any specific ambitious climate policies, we also considered scenarios that achieve the Paris Agreement goals. The goals of maintaining global temperature increase by 2100 below $2{ }^{\circ} \mathrm{C}$ or $1.5^{\circ} \mathrm{C}$ have frequently been modelled in the IAM community ${ }^{55}$ by imposing global or regional carbon prices on greenhouse gas emissions throughout the decades. Another common approach in optimization models like MESSAGEix-GLOBIOM is to impose a cumulative carbon budget and let the model find economically optimal mitigation strategies. For this analysis, we combined these approaches as described in Rogelj et al. ${ }^{56}$ to produce scenarios that meet predefined carbon budgets $\left(550 \mathrm{GtCO}_{2}\right.$ and $1,000 \mathrm{GtCO}_{2}$ for $1.5^{\circ} \mathrm{C}$ and below $2{ }^{\circ} \mathrm{C}$ scenarios respectively) until reaching net-zero emissions by mid-century, while staying at net-zero $\mathrm{CO}_{2}$ emissions afterwards. These scenarios are modelled as a combination of carbon prices and constraints on emissions and are independent of the COVID-19-related assumptions. This scenario set-up allows us to combine climate mitigation targets with different post-pandemic recovery pathways and to compare the latter from different perspectives. In addition, the restore and green push scenarios were run with the carbon price that is consistent with the $1.5^{\circ} \mathrm{C}$ target (equivalent to the mitigation scenario restore with $550 \mathrm{GtCO}_{2}$ budget). This allows studying the effect on emissions of different energy demand trajectories given the exact same carbon cost assumptions, to supplement the set-up that explores the economic differences while maintaining the same carbon emission goals.

\section{Data availability}

All data sources used for this study are cited in the Supplementary Information. Data are also available from the corresponding author upon request. The results presented in this article explore only a small portion of the model outputs from our scenario analysis. A web tool hosted by IIASA provides access to a database of these and more variables of interest, defined for each scenario on the detail of MESSAGE regions, with a few example workspaces available within the ENGAGE Scenario Explorer at https://data.ene.iiasa.ac.at/engage/\#/workspaces/60. The Scenario Explorer is a versatile open access tool to browse, visualize and download data and results. Users can freely create a private workspace where customized plots can be saved and shared.

For tutorials on how to use the Scenario Explorer, please visit https://software.ene. iiasa.ac.at/ixmp-server/tutorials.html.

SR1.5 scenarios ${ }^{55}$ have been made available through Huppmann et al..$^{57}$ and Huppmann et al. ${ }^{58}$ at https://data.ene.iiasa.ac.at/iamc-1.5c-explorer/.

\section{Code availability}

Model code has been published open source at https://github.com/iiasa/message ix, with online documentation available at https://docs.messageix.org/en/stable/ and in Krey et al..$^{30}$. The code and data used to generate the figures in the main text are made available at https://github.com/iiasa/covid-energy-demand-scenarios and https://doi.org/10.5281/zenodo.5211169 $9^{59}$, respectively.

Received: 25 January 2021; Accepted: 4 August 2021; Published online: 11 October 2021

\section{References}

1. van Ballegooijen, H., Goossens, L., Bruin, R. H., Michels, R. \& Krol, M. Concerns, quality of life, access to care and productivity of the general population during the first 8 weeks of the coronavirus lockdown in Belgium and the Netherlands. BMC Health Serv. Res. 21, 227 (2021).

2. Fernandes, N. Economic effects of coronavirus outbreak (COVID-19) on the world economy. SSRN Electron. J. https://doi.org/10.2139/ssrn.3557504 (2020)

3. Nicola, M. et al. The socio-economic implications of the coronavirus pandemic (COVID-19): a review. Int. J. Surg. 78, 185-193 (2020).

4. Forster, P. M. et al. Current and future global climate impacts resulting from COVID-19. Nat. Clim. Change 10, 913-919 (2020).

5. Jiang, P., Van Fan, Y. \& Klemeš, J. J. Impacts of COVID-19 on energy demand and consumption: challenges, lessons and emerging opportunities. Appl. Energy 285, 116441 (2021).

6. Le Quéré, C. et al. Temporary reduction in daily global $\mathrm{CO}_{2}$ emissions during the COVID-19 forced confinement. Nat. Clim. Change 10, 647-653 (2020).

7. Liu, Z. et al. Near-real-time monitoring of global $\mathrm{CO}_{2}$ emissions reveals the effects of the COVID-19 pandemic. Nat. Commun. 11, 5172 (2020).
8. Friedlingstein, P. et al. Global carbon budget 2020. Earth Syst. Sci. Data 12, 3269-3340 (2020).

9. Sharmina, M. et al. Decarbonising the critical sectors of aviation, shipping, road freight and industry to limit warming to $1.5-2^{\circ} \mathrm{C}$. Clim. Policy 21, 455-474 (2020)

10. Andrijevic, M., Schleussner, C.-F., Gidden, M. J., McCollum, D. L. \& Rogelj, J. COVID-19 recovery funds dwarf clean energy investment needs. Science 370, 298-300 (2020).

11. Pianta, S., Brutschin, E., van Ruijven, B. \& Bosetti, V. Faster or slower decarbonization? Policymaker and stakeholder expectations on the effect of the COVID-19 pandemic on the global energy transition. Energy Res. Soc. Sci. 76, 102025 (2021).

12. Hepburn, C., O'Callaghan, B., Stern, N., Stiglitz, J. \& Zenghelis, D. Will COVID-19 fiscal recovery packages accelerate or retard progress on climate change? Oxf. Rev. Econ. Policy 36, S359-S381 (2020).

13. Creutzig, F. et al. Towards demand-side solutions for mitigating climate change. Nat. Clim. Change 8, 268-271 (2018).

14. Creutzig, F. et al. Beyond technology: demand-side solutions for climate change mitigation. Annu. Rev. Environ. Resour. 41, 173-198 (2016).

15. Dafnomilis, I. et al. Exploring the Impact of the COVID-19 Pandemic on Global Emission Projections: Assessment of Green Versus Non-Green Recovery (NewClimate Institute, 2020).

16. Malliet, P., Reynès, F., Landa, G., Hamdi-Cherif, M. \& Saussay, A. Assessing short-term and long-term economic and environmental effects of the COVID-19 crisis in France. Environ. Resour. Econ. 76, 867-883 (2020).

17. Keramidas, K. et al. Global Energy and Climate Outlook 2020: A New Normal Beyond COVID-19 (JRC Science for Policy Report, 2021).

18. Bertram, C. et al. COVID-19-induced low power demand and market forces starkly reduce $\mathrm{CO}_{2}$ emissions. Nat. Clim. Change 11, 193-196 (2021).

19. Lahcen, B. et al. Green recovery policies for the COVID-19 crisis: modelling the impact on the economy and greenhouse gas emissions. Environ. Resour. Econ. 76, 731-750 (2020).

20. Huppmann, D. et al. The MESSAGEix integrated assessment model and the ix modeling platform (ixmp): an open framework for integrated and cross-cutting analysis of energy, climate, the environment, and sustainable development. Environ. Model. Softw. 112, 143-156 (2019).

21. Energy Efficiency 2020 (International Energy Agency, 2020).

22. Madurai Elavarasan, R. et al. COVID-19: impact analysis and recommendations for power sector operation. Appl. Energy 279, 115739 (2020).

23. Hook, A., Court, V., Sovacool, B. K. \& Sorrell, S. A systematic review of the energy and climate impacts of teleworking. Environ. Res. Lett. 15, 093003 (2020)

24. Grubler, A. et al. A low energy demand scenario for meeting the $1.5^{\circ} \mathrm{C}$ target and sustainable development goals without negative emission technologies. Nat. Energy 3, 515-527 (2018).

25. Rogelj, J., Forster, P. M., Kriegler, E., Smith, C. J. \& Séférian, R. Estimating and tracking the remaining carbon budget for stringent climate targets. Nature 571, 335-342 (2019).

26. Global EV Outlook 2020 (International Energy Agency, 2020).

27. Global Energy Review 2020: The Impacts of the COVID-19 Crisis on Global Energy Demand and $\mathrm{CO}_{2}$ Emissions (International Energy Agency, 2020).

28. IPCC Special Report on Global Warming of $1.5^{\circ} \mathrm{C}$ (eds Masson-Delmotte, V. et al.) (WMO, 2018).

29. Mengis, N. \& Matthews, H. D. Non- $\mathrm{CO}_{2}$ forcing changes will likely decrease the remaining carbon budget for $1.5^{\circ} \mathrm{C}$. npj Clim. Atmos. Sci. 3, 19 (2020).

30. Krey, V. et al. MESSAGEix-GLOBIOM Documentation - 2020 Release (International Institute for Applied Systems Analysis, 2020).

31. Messner, S. \& Schrattenholzer, L. MESSAGE-MACRO: linking an energy supply model with a macroeconomic module and solving it iteratively. Energy 25, 267-282 (2000).

32. Fricko, O. et al. The marker quantification of the Shared Socioeconomic Pathway 2: a middle-of-the-road scenario for the 21st century. Glob. Environ. Change 42, 251-267 (2017).

33. Riahi, K. et al. The shared socioeconomic pathways and their energy, land use, and greenhouse gas emissions implications: an overview. Glob. Environ. Change 42, 153-168 (2017).

34. Rogelj, J. et al. Energy system transformations for limiting end-of-century warming to below $1.5^{\circ} \mathrm{C}$. Nat. Clim. Change 5, 519-527 (2015).

35. McCollum, D. L. et al. Energy investment needs for fulfilling the Paris agreement and achieving the sustainable development goals. Nat. Energy 3, 589-599 (2018)

36. Riahi, K. et al. in Global Energy Assessment: Toward a Sustainable Future (GEA Writing Team) Ch. 17 (Cambridge Univ. Press, 2012).

37. The World in 2050 Initiative Innovations for Sustainability: Pathways to an Efficient and Post-pandemic Future (International Institute for Applied Systems Analysis, 2020).

38. O'Neill, B. C. et al. The roads ahead: narratives for shared socioeconomic pathways describing world futures in the 21st century. Glob. Environ. Change 42, 169-180 (2017). 
39. Apple LLC Mobility Trends Reports (Apple, accessed March 2021); https://covid19.apple.com/mobility

40. Google LLC Community Mobility Reports (Google, accessed March 2021); https://www.google.com/covid19/mobility

41. Altig, D. et al. Economic uncertainty before and during the COVID-19 pandemic. J. Public Econ. 191, 104274 (2020).

42. Global Economic Prospects, January 2020: Slow Growth, Policy Challenges (The World Bank, 2020).

43. Global Outlook: Pandemic, Recession: The Global Economy in Crisis (The World Bank, 2020).

44. Estrada, F., Tol, R. S. J. \& Gay-García, C. The persistence of shocks in GDP and the estimation of the potential economic costs of climate change. Environ. Model. Softw. 69, 155-165 (2015).

45. Parkinson, J. Coronavirus: Traffic 'reaching early 1970s levels'. BBC News (5 May 2020).

46. GEP Impact of COVID-19 on the medical supply chain. Supply Chain Blog https://www.gep.com/blog/mind/impact-of-covid-19-outbreak-on-medi cal-supply-chain-from-china-to-global (2020).

47. Global Disruption: The Impact of COVID-19 on the Bioindustry Sector (AFRY, 2020).

48. Preparing the Chemicals Industry for 'the Day After' the COVID-19 Pandemic (PwC, 2020).

49. Huuhka, S. Vacant residential buildings as potential reserves: a geographical and statistical study. Build. Res. Inf. 44, 816-839 (2016).

50. Vacancy rates of office space in selected cities worldwide in 2018 and 2021. Statista https://www.statista.com/statistics/978457/ vacancy-rates-office-space-cities-worldwide (2021).

51. O'Brien, W. et al. An international review of occupant-related aspects of building energy codes and standards. Build. Environ. 179, 106906 (2020).

52. O’Brien, W. \& Yazdani Aliabadi, F. Does telecommuting save energy? A critical review of quantitative studies and their research methods. Energy Build. 225, 110298 (2020).

53. Hale, T. et al. A global panel database of pandemic policies (Oxford COVID-19 Government Response Tracker). Nat. Hum. Behav. 5, 529-538 (2020).

54. Working from Home: Estimating the Worldwide Potential International Labour Organization Policy Brief (International Labour Organization, 2020).

55. Rogelj, J. et al. in IPCC Special Report on Global Warning of $1.5^{\circ} \mathrm{C}$ (eds Masson-Delmotte V. et al.) (WMO, 2018).

56. Rogelj, J. et al. A new scenario logic for the Paris Agreement long-term temperature goal. Nature 573, 357-363 (2019).

57. Huppmann, D. et al. IAMC $1.5^{\circ} \mathrm{C}$ scenario explorer and data hosted by IIASA. Zenodo https://doi.org/10.5281/ZENODO.3363345 (2019).
58. Huppmann, D., Rogelj, J., Kriegler, E., Krey, V. \& Riahi, K. A new scenario resource for integrated $1.5^{\circ} \mathrm{C}$ research. Nat. Clim. Change 8 , 1027-1030 (2018)

59. Kikstra, J. S. et al. Climate mitigation scenarios with persistent COVID-19 related energy demand changes. Nat. Energy https://doi.org/10.5281/ zenodo.5211169 (2021).

\section{Acknowledgements}

This study was funded by European Union's Horizon 2020 research and innovation programme under grant agreement no. 821471 (ENGAGE) (J.S.K., A.V., B.B.-K., F.L., B.v.R., J.R., B.Z., O.F. and K.R.). This project received funding from the European Union's Horizon 2020 research and innovation programme under grant agreement no. 821124 (NAVIGATE) (F.L., B.v.R., C.W. and K.R.). This work was supported by the Natural Environment Research Council under grant agreement no. NE/S007 415/1 (J.S.K.)

\section{Author contributions}

J.S.K. and A.V. coordinated the study, performed and analysed the model runs and made the visualizations. B.B.-K. and F.L. performed the bottom-up energy activity and structural change analysis. F.L., B.B.-K., J.S.K., A.V. and B.v.R. designed and analysed the energy demand pathways. B.Z. and O.F. contributed to modelling and scenario runs J.R. and C.W. designed the scenario typology and mitigation pathway selection. K.R. conceived the study. All authors contributed to writing and reviewing the manuscript and analysis.

\section{Competing interests}

The authors declare no competing interests.

\section{Additional information}

Supplementary information The online version contains supplementary material available at https://doi.org/10.1038/s41560-021-00904-8.

Correspondence and requests for materials should be addressed to Jarmo S. Kikstra. Peer review information Nature Energy thanks Florian Knobloch and the other, anonymous, reviewer(s) for their contribution to the peer review of this work.

Reprints and permissions information is available at www.nature.com/reprints. Publisher's note Springer Nature remains neutral with regard to jurisdictional claims in published maps and institutional affiliations.

(c) The Author(s), under exclusive licence to Springer Nature Limited 2021 